\title{
Tratamento da osteonecrose dos maxilares associada ao uso de medicamentos -
}

\section{revisão de literatura}

Treatment of medication-related osteonecrosis of the jaw - literature review

Tratamiento de la osteonecrosis mandibular relacionada con medicamentos - revisión de literatura

\section{Resumo}

Introdução: A osteonecrose dos maxilares associada ao uso de medicamentos é uma doença que se dá pelo uso de terapias com drogas antirreabsortivas, como bisfosfonatos e Denosumab, ou antiangiogênicos. Embora a manifestação desta doença não tenha alta incidência, sua ocorrência gera um impacto significativo na vida do paciente, levando à sintomatologia dolorosa, com supuração e odor fétido. Apesar dos vários tratamentos empregados, não existe um consenso acerca da maior eficácia de um tratamento frente a outro. Assim, este trabalho objetiva demonstrar as diferentes modalidades de tratamento e os benefícios aliados a cada uma. Materiais e Métodos: Buscas feitas na base de dados PubMed/MEDLINE sobre o tema Osteonecrose dos Maxilares Associada ao Uso de Medicamentos, aliado a operadores boleanos, durante os meses Maio, Junho e Julho de 2020. Resultados: A pesquisa resultou em 143 artigos e, após a aplicação dos critérios de elegibilidade, 49 foram selecionados (23 estudos prospectivos, 17 retrospectivos e 9 séries de caso), englobando 2617 pacientes e 2848 lesões. Os tratamentos foram divididos em 5 modalidades (terapias adjuvantes, tratamentos cirúrgicos agressivos, terapias teciduais, tratamento assistido por medicamentos e terapias cirúrgicas conservadoras).Conclusão: Vários tratamentos são propostos e parece claro que as cirurgias agressivas são as terapias com melhores resultados, sobretudo quando aliadas a uma terapia tecidual ou adjuvante. As cirurgias conservadoras apresentam papel importante, principalmente, em pacientes portadores de condições sistêmicas que contraindiquem as cirurgias maiores.

Palavras-chave: Osteonecrose da arcada osseodentária associada a difosfonatos; Osteonecrose; Assistência odontológica.

\begin{abstract}
Introduction: Osteonecrosis of the jaws associated with the use of medications is a disease that occurs through the use of therapies with antiresorptive drugs, such as bisphosphonates and Denosumab, or antiangiogenics. Although the manifestation of this disease does not have a high incidence, its occurrence generates a significant impact on the patient's life, leading to painful symptoms, with suppuration and a foul odor. Despite the various treatments used, there is no consensus on the greater effectiveness of one treatment compared to another. Thus, this work aims to demonstrate the different treatment modalities and the benefits allied to each one. Materials and Methods: Searches made in the PubMed / MEDLINE database on the theme Osteonecrosis of the Jaws Associated with the Use of Medicines, together with Boolean operators, during May, June and July 2020. Results: The search resulted in 143 articles and, after applying the eligibility criteria, 49 were selected (23 prospective studies, 17 retrospective and 9 case series), comprising 2617 patients and 2848 injuries. The treatments were divided into 5 modalities (adjuvant therapies, aggressive surgical treatments, tissue therapies, medication-assisted treatment and conservative surgical therapies). Conclusion: Several treatments are proposed and it seems clear that aggressive surgeries are the therapies with the best results, especially when combined with tissue or adjuvant therapy. Conservative surgeries play an important role, especially in patients with systemic conditions that contraindicate major surgeries.
\end{abstract}


Keywords: Bisphosphonate-associated osteonecrosis of the jaw; Osteonecrosis; Dental care.

\section{Resumen}

Introducción: La osteonecrosis de los maxilares asociada al uso de medicamentos es una enfermedad que se presenta por el uso de terapias con fármacos antirresortivos, como bifosfonatos y denosumab, o antiangiogénicos. Si bien la manifestación de esta enfermedad no tiene una alta incidencia, su aparición genera un impacto significativo en la vida del paciente, provocando síntomas dolorosos, con supuración y mal olor. A pesar de los diversos tratamientos utilizados, no existe consenso sobre la mayor efectividad de un tratamiento en comparación con otro. Así, este trabajo tiene como objetivo demostrar las diferentes modalidades de tratamiento y los beneficios aliados a cada uno. Materiales y Métodos: Búsquedas realizadas en la base de datos PubMed / MEDLINE sobre el tema Osteonecrosis de la mandíbula asociada al uso de medicamentos, junto con operadores booleanos, durante mayo, junio y julio de 2020. Resultados: La búsqueda resultó en 143 artículos y, después aplicando los criterios de elegibilidad, se seleccionaron 49 (23 estudios prospectivos, 17 retrospectivos y 9 series de casos), que comprenden 2617 pacientes y 2848 lesiones. Los tratamientos se dividieron en 5 modalidades (terapias adyuvantes, tratamientos quirúrgicos agresivos, terapias tisulares, tratamiento asistido por medicamentos y terapias quirúrgicas conservadoras). Conclusión: Se proponen varios tratamientos y parece claro que las cirugías agresivas son las terapias con mejores resultados, especialmente cuando se combinan con terapia tisular o adyuvante. Las cirugías conservadoras juegan un papel importante, especialmente en pacientes con condiciones sistémicas que contraindican cirugías mayores.

Palabras clave: Osteonecrosis de los maxilares asociada a difosfonatos; Osteonecrosis; Atención odontológica.

\section{Introdução}

A osteonecrose dos maxilares é uma condição patológica que se desenvolve por alterações no metabolismo ósseo e afeta negativamente a qualidade de vida dos pacientes acometidos, causando incômodos e morbidades (Ruggiero et al., 2009). Estudos iniciais demonstraram que os bisfosfonatos são capazes de interferir no metabolismo ósseo, sendo fármacos padrãoouro para determinadas doenças, como osteonecrose e metástases ósseas, porém demonstram como efeito adverso a possibilidade do desenvolvimento de um quadro de osteonecrose nos ossos maxilares (Ruggiero et al., 2009; AdvisoryTask Force on Bisphosphonate-Related Ostenonecrosis of the Jaws, American Association of Oral and Maxillofacial Surgeons, 2007). No ano de 2014, Ruggiero et al.(2014) recomendaram que o antigo termo "Osteonecrose dos Maxilares Induzida por Bisfosfonatos" (ONIB) fosse alterado para “Osteonecrose dos Maxilares Induzida por Medicamentos” (ONMIM), visto que fármacos que não fazem parte do grupo dos bisfosfonatos, como os antiangiogênicos, medicamentos antiresorptivos e Denosumab, medicamentos usados para prevenir fraturas ósseas associadas à osteoporose e bloquear o desenvolvimento de metástases associadas a doenças cancerígenas, demonstraram capacidade de desencadear episódios de osteonecrose dos maxilares (Goldvaser \& Amir, 2019; Rodan \& Fleisch, 1996; Watts, 2003; Papapoulos et al., 2012; Fizazi et al., 2011).

Tratamentos com bisfosfonatos endovenosos apresentam incidência de ONMIM entre 0,8\% e 12\%, já os tratamentos orais apresentam incidência inferior a $1 \%$ (Ruggiero et al., 2009). A incidência de ONMIM em pacientes sob uso de Denosumab varia entre 0,04\% e 1,9\%, a depender da dose utilizada (Ruggiero et al., 2009; Papapoulos et al., 2012; Qi, Tang, He, Yao \& Shen, 2014). Para os agentes inibidores de angiogênese, Guarneri et al.(2010) relataram em um estudo que o risco de desenvolver osteonecrose dos maxilares associada ao seu uso é de $0,2 \%$.

A ONMIM é caracterizada por meio de estadiamento das lesões, sendo que lesões mais simples, com características menos pronunciadas, e que apresentam melhores prognósticos são caracterizadas em estágio 0. À medida que a lesão evolui e se prognóstico torna-se pior, o estágio se eleva, sendo que as lesões mais agressivas são caracterizadas como estágio 3 (Ruggiero et al., 2014).

O tratamento para a osteonecrose dos maxilares induzida por medicamentos é um desafio. Diversas modalidades de tratamento foram propostas, desde técnicas conservadoras, por meio de enxaguantes bucais e antibióticos, até ressecções totais, passando ainda por terapias teciduais e técnicas adjuvantes (Giudice et al., 2018; Favia, Tempesta, Limongelli, Crincoli \& Maiorano, 2016; Rotaru, Kim, Kim \& Park, 2015; Karasneh, Al-Eryani, Clark \& Sedghizadeh, 2016; Thumbigere-Math et al., 
2009). É válido analisar que muitos tratamentos podem atuar como uma via de mão-dupla, ou seja, curando o paciente da osteonecrose, porém colocando-o em uma situação também problemática, que pode afetar negativamente sua vida.

Diante de tantos tratamentos e sabendo o quanto a ONMIM afeta negativamente a vida do paciente, este trabalho fezse necessário para avaliar as diversas formas de tratamento propostas, bem como seus resultados e possíveis consequências. O objetivo deste trabalho é avaliaras diferentes técnicas de tratamento propostas, comparando seus resultados alcançados.

\section{Metodologia}

O presente estudo é uma revisão de literatura, de caráter descritivo e exploratório que reúne resultados de estudos previamente publicados acerca do tema em questão, possibilitando a ampliação dos conhecimentos sobre a temática (Pereira, Shitsuka, Parreira \& Shitsuka, 2018)

O estudo bibliográfico foi realizado a partir de buscas feitas na base de dados PubMed/MEDLINE sobre o tema Osteonecrose dos Maxilares Induzida por Medicamentos, durante os meses Maio, Junho e Julho de 2020, com pesquisas avançadas, utilizando termos específicos e operadores boleanos. Foi aplicado o filtro "Clinical Trial” para as pesquisas. As combinações de termos com operadores boleanos utilizados na pesquisa foram: "Medication related osteonecrosis of the jaw" AND "treatment"; "medication related osteonecrosis of the jaw" AND "therapy"; "bisphosphonate related osteonecrosis of the jaw" AND "treatment"; "bisphosphonate related osteonecrosis of the jaw" AND "therapy"; "denosumab" AND "osteonecrosis" AND "treatment"; "denosumab" AND "osteonecrosis" AND "therapy"; "medication associated osteonecrosis of the jaw" AND "treatment"; "medication associated osteonecrosis of the jaw" AND "therapy" "medication associated osteonecrosis of the jaw" AND "management" "bisphosphonate associated osteonecrosis of the jaw" AND "treatment"; "bisphosphonate associated osteonecrosis of the jaw" AND "therapy" "bisphosphonate associated osteonecrosis of the jaw" AND "management"; "medication related osteonecrosis of the jaw" AND "management"; "bisphosphonate related osteonecrosis of the jaw" AND “management" "denosumab" AND “osteonecrosis" AND “management".

Os critérios de inclusão utilizados foram artigos que apresentaram conteúdos disponíveis em inglês ou português; estudos de pesquisa retrospectiva e prospectiva relacionados ao tema; série de casos clínicos que abordaram o tema. Os critérios de exclusão empregados foram estudos laboratoriais, estudos em animais, revisões de literatura, revisões sistemáticas e relatos de casos que apresentassem menos de três casos.

Após a busca pelos artigos na base de dados, a primeira fase de seleção foi realizada a partir da leitura dos títulos, eliminando os artigos que apresentassem títulos não relacionados ao tema, bem como artigos duplicados. A segunda etapa de seleção foi realizada a partir da leitura dos resumos; e a terceira a partir da leitura do artigo na íntegra. A seleção dos estudos, sua leitura e avaliação foram realizadas por dois pesquisadores. A avaliação foi conduzida baseada na extração dos seguintes dados: número de lesões de osteonecrose, número de pacientes acometidos, média de idade dos pacientes, sexo dos pacientes, período médio de uso da medicação, motivo do uso da medicação, osso afetado pelas lesões, estágio das lesões, tempo de acompanhamento do paciente, tratamento empregado e os resultados finais obtidos.

\section{Resultados}

A estratégia de busca localizou 143 publicações. Após a exclusão dos artigos cuja temática não contemplava nossa abordagem (83), repetidas (10) ou que não se encaixavam nos critérios de inclusão (1), foram selecionadas 49 publicações (Giudice et al., 2018; Favia et al., 2016; Rotaru et al., 2015; Karasneh et al., 2016; Thumbigere-Math et al., 2009; Ripamonti, Cislaghi, Mariani \& Maniezzo, 2011; Jung et al., 2017; Kwon, Lee, Choi, Lee \& Kim, 2012; Laimer et al., 2017; Bedogni et al., 2011; Stockmann et al., 2010; Rugani et al., 2015; Hallmer et al., 2018; Reich, Bilkenroth, Schubert, Wickenhauser \& 
Eckert, 2015; Mauceri et al., 2018; Inchingolo et al., 2017; Mourão et al., 2020; Calvani et al., 2018; Lemound et al., 2018; Coviello et al., 2012; Park, Kim \& Kim, 2017; Ikeda et al., 2015; Nisi et al., 2018; Lee, Hsiao \& Chen, 2014; Moretti, Pelliccioni, Montebugnoli \& Marchetti, 2011; Fortuna et al., 2012; Freiberger et al., 2012; Ristow et al., 2017; Agrillo et al., 2012; Atalay et al., 2011; Favia, Tempesta, Limongelli, Crincoli \& Maiorano, 2018; Pichardo, Kuijpers \& van Merkesteyn, 2016; Voss et al., 2012; El-Rabbany, Lam, Shah \& Azarpazhooh, 2019; Lopes, Rabelo, Rocha, Carvalho \& Alves, 2015; Kim et al., 2017; Hoefert et al., 2017; Eguchi et al., 2017; Ruggiero \& Kohn, 2015; Owosho, Estilo, Huryn \& Yom, 2016; Lerman et al., 2013; Coropciuc et al., 2017; Stübinger et al., 2009; Voss et al., 2017; Aljohani et al., 2018; Pichardo \& van Merkesteyn, 2016; Aljohani et al., 2019; Mozzati, Gallesio, Arata, Pol \& Scoletta, 2012; Curi et al., 2011). Dentre estes, 23 foram estudos prospectivos (Giudice et al., 2018; Thumbigere-Math et al., 2009; Ripamonti et al., 2011; Jung et al., 2017; Kwon et al., 2012; Laimer et al., 2017; Bedogni et al., 2011; Stockmann et al., 2010; Rugani et al., 2015; Hallmer et al., 2018; Reich et al. 2015; Mauceri et al., 2018; Inchingolo et al., 2017; Mourão et al., 2020; Calvani et al., 2018; Lemound et al., 2018; Coviello et al., 2012; Park et al., 2017; Ikeda et al., 2015; Nisi et al., 2018; Lee et al., 2014; Moretti et al., 2011; Fortuna et al., 2012), 17 foram estudos retrospectivos (Favia et al., 2016; Freiberger et al., 2012; Ristow et al., 2017; Agrillo et al., 2012; Atalay et al., 2011; Favia et al., 2018; Pichardo et al., 2016; Voss et al., 2012; El-Rabbany et al., 2019; Lopes et al., 2015; Kim et al., 2017; Hoefert et al., 2017; Eguchi et al., 2017; Ruggiero \& Kohn, 2015; Owosho et al., 2016; Lerman et al., 2013; Coropciuc et al., 2017) e 9 foram estudos no formato de série de casos (Rotaru et al., 2015; Karasneh et al., 2016; Stübinger et al., 2009; Voss et al., 2017; Aljohani et al., 2018; Pichardo \& van Merkesteyn, 2016; Aljohani et al., 2019; Mozzati et al., 2012; Curi et al., 2011). Ao todo, as publicações selecionadas abordaram 2848 lesões, em 2617 pacientes, com média de idade de 63,06 anos (Tabela 1).

Tabela 1. Características dos Pacientes.

\begin{tabular}{|c|c|c|c|c|c|}
\hline & $\begin{array}{ll}\mathrm{N}^{\mathrm{o}} & \mathrm{de} \\
\text { lesões } & \end{array}$ & $\begin{array}{ll}\mathrm{N}^{\mathrm{o}} & \mathrm{de} \\
\text { pacientes } & \end{array}$ & $\mathrm{N}^{\circ}$ homens & $\mathrm{N}^{\circ}$ mulheres & $\begin{array}{lr}\text { Média de } \\
\text { idade dos } \\
\text { pacientes }\end{array}$ \\
\hline $\begin{array}{l}\text { Tratamento Assistido por Medicamento } \\
\text { (Owosho et al., 2016; Ikeda et al., 2015; } \\
\text { Karasneh et al. 2016) }\end{array}$ & 39 & 32 & 18 & 14 & 72,1 anos \\
\hline $\begin{array}{l}\text { Terapias Cirúrgicas Conservadoras } \\
\text { (Lerman et al., 2013; Coropciuc et al., 2017; } \\
\text { Nisi et al., 2018; Lee et al., 2014; Moretti et } \\
\text { al., 2011; Fortuna et al., 2012; } \\
\text { Thumbigere-Math } \text { et al., 2009) }\end{array}$ & 426 & 378 & 151 & 227 & 48,18 anos \\
\hline $\begin{array}{l}\text { Terapias Teciduais (Mauceri et al., 2018; } \\
\text { Inchingolo et al., 2017; Mourão et al., 2020; } \\
\text { Calvani et al., 2018; Lemound et al., 2018; } \\
\text { Coviello et al., 2012; Park et al., 2017; Jung } \\
\text { et al., 2017; Aljohani et al., 2019; Mozzati et } \\
\text { al., 2012; Curi et al., 2011; Rotaru et al., } \\
\text { 2015) }\end{array}$ & 254 & 248 & 80 & 240 & 66,85 anos \\
\hline $\begin{array}{l}\text { Terapias Adjuvantes (Freiberger et al., } \\
\text { 2012; Ristow et al., 2017; } \\
\text { Agrillo et al., 2012; Atalay et al., 2011; } \\
\text { Ripamonti } \text { et al., 2011; Giudice } \text { et al., 2018; } \\
\text { Kwon } \text { et al., 2012; } \\
\text { Laimer } \text { et al., 2017; } \\
\text { Bedogni } \text { et al., 2011; Stübinger } \text { et al., 2009; } \\
\text { Voss } \text { et al., 2017) }\end{array}$ & 332 & 302 & 110 & 192 & 66,45 anos \\
\hline
\end{tabular}




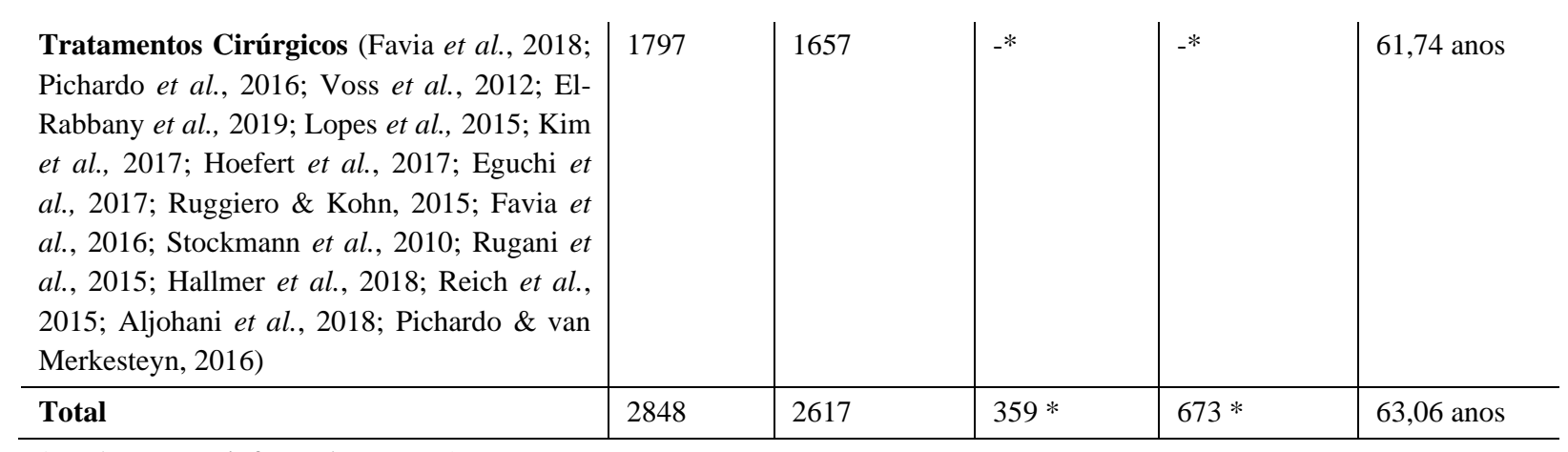

*- Número não informado Fonte: Autores.

Observou-se que o maxilar mais acometido pela ONMIM é a mandíbula. As lesões estavam presentes na maxila em 725 pacientes, na mandíbula em 1817 pacientes e em ambos os maxilares em 98 pacientes (Giudice et al., 2018; Favia et al., 2016; Rotaru et al., 2015; Karasneh et al., 2016; Thumbigere-Math et al., 2009; Ripamonti et al., 2011; Kwon et al., 2012; Laimer et al., 2017; Bedogni et al., 2011; Stockmann et al., 2010; Hallmer et al., 2018; Reich et al. 2015; Mauceri et al., 2018; Mourão et al., 2020; Calvani et al., 2018; Lemound et al., 2018; Coviello et al., 2012; Park et al., 2017; Ikeda et al., 2015; Nisi et al., 2018; Lee et al., 2014; Moretti et al., 2011; Fortuna et al., 2012; Ristow et al., 2017; Agrillo et al., 2012; Atalay et al., 2011; Favia et al., 2018; Pichardo et al., 2016; Voss et al., 2012; El-Rabbany et al., 2019; Lopes et al., 2015; Kim et al., 2017; Hoefert et al., 2017; Eguchi et al., 2017; Ruggiero \& Kohn, 2015; Owosho et al., 2016; Lerman et al., 2013; Coropciuc et al., 2017; Stübinger et al., 2009; Voss et al., 2017; Aljohani et al., 2018; Pichardo \& van Merkesteyn, 2016; Aljohani et al., 2019; Mozzati et al., 2012; Curi et al., 2011). Quanto ao estágio da lesão, 23 caracterizavam-se como estágio 0, 348 como estágio 1, 1261 como estágio 2 e 809 como estágio 3 (Giudice et al., 2018; Favia et al., 2016; Rotaru et al., 2015; Karasneh et al., 2016; Thumbigere-Math et al., 2009; Jung et al., 2017; Kwon et al., 2012; Laimer et al., 2017; Bedogni et al., 2011; Stockmann et al., 2010; Rugani et al., 2015; Hallmer et al., 2018; Reich et al. 2015; Mourão et al., 2020; Calvani et al., 2018; Lemound et al., 2018; Park et al., 2017; Ikeda et al., 2015; Nisi et al., 2018; Lee et al., 2014; Fortuna et al., 2012; Ristow et al., 2017; Atalay et al., 2011; Favia et al., 2018; Voss et al., 2012; El-Rabbany et al., 2019; Lopes et al., 2015; Kim et al., 2017; Hoefert et al., 2017; Eguchi et al., 2017; Ruggiero \& Kohn, 2015; Owosho et al., 2016; Lerman et al., 2013; Coropciuc et al., 2017; Voss et al., 2017; Aljohani et al., 2018; Pichardo \& van Merkesteyn, 2016; Aljohani et al., 2019; Curi et al., 2011).

A maioria dos pacientes fazia utilização do medicamento por motivos de neoplasias de mama com metástases ósseas. Diversos autores (Bedogni et al., 2011; Coviello et al., 2012; Lee et al., 2014; Moretti et al., 2011; Pichardo et al., 2016) relataram pacientes que utilizavam a medicação para tratamento de Mieloma Múltiplo. O principal regime terapêutico relatado envolveu o uso de Zolendronato por via intravenosa (Giudice et al., 2018; Favia et al., 2016; Thumbigere-Math et al., 2009; Laimer et al., 2017; Rugani et al., 2015; Hallmer et al., 2018; Reich et al. 2015; Mourão et al., 2020; Calvani et al., 2018; Nisi et al., 2018; Freiberger et al., 2012; Coropciuc et al., 2017; Voss et al., 2017; Aljohani et al., 2018; Pichardo \& van Merkesteyn, 2016; Aljohani et al., 2019; Mozzati et al., 2012).

As modalidades terapêuticas para a ONMIM foram divididas em cinco grupos: tratamentos assistidos por medicamento, tratamentos cirúrgicos conservadores, terapias teciduais, terapias adjuvantes e tratamentos cirúrgicos agressivos. (Tabela 2). 
Tabela 2. Características dos tipos de terapia.

\begin{tabular}{|c|c|c|c|}
\hline & Estudos Retrospectivos & Estudos Prospectivos & Série de Casos \\
\hline $\begin{array}{l}\text { Tratamento Assistido por } \\
\text { Medicamentos }\end{array}$ & Owosho et al. (2016) & Ikeda et al. (2015) & Karasneh et al. 2016 \\
\hline $\begin{array}{ll}\text { Terapias } & \text { Cirúrgicas } \\
\text { Conservadoras } & \end{array}$ & $\begin{array}{l}\text { Lerman et al. (2013) } \\
\text { Coropciuc et al. (2017) }\end{array}$ & $\begin{array}{l}\text { Nisi et al. (2018) } \\
\text { Lee et al. (2014) } \\
\text { Moretti et al. (2011) } \\
\text { Fortuna et al. (2012) } \\
\text { Thumbigere-Math } \text { et al. (2009) }\end{array}$ & - \\
\hline Terapias Teciduais & - & $\begin{array}{l}\text { Mauceri et al. (2018) } \\
\text { Inchingolo et al. (2017) } \\
\text { Mourão et al. (2020) } \\
\text { Calvani et al. (2018) } \\
\text { Lemound } \text { et al. (2018) } \\
\text { Coviello et al. (2012) } \\
\text { Park et al. (2017) } \\
\text { Jung et al. (2017) }\end{array}$ & $\begin{array}{l}\text { Aljohani et al. (2019) } \\
\text { Mozzati et al. (2012) } \\
\text { Curi et al. (2011) } \\
\text { Rotaru et al. (2015) }\end{array}$ \\
\hline Terapias Adjuvantes & $\begin{array}{l}\text { Freiberger } \text { et al. (2012) } \\
\text { Ristow et al. (2017) } \\
\text { Agrillo et al. (2012) } \\
\text { Atalay et al. (2011) }\end{array}$ & $\begin{array}{l}\text { Ripamonti et al. (2011) } \\
\text { Giudice } \text { et al. }(2018) \\
\text { Kwon } \text { et al. } \text { (2012) } \\
\text { Laimer } \text { et al.(2017) } \\
\text { Bedogni et al. }(2011)\end{array}$ & $\begin{array}{l}\text { Stübinger } \text { et al. } \\
\text { (2009) } \\
\text { Voss et al. (2017) }\end{array}$ \\
\hline $\begin{array}{l}\text { Tratamentos } \quad \text { Cirúrgicos } \\
\text { Agressivos }\end{array}$ & $\begin{array}{l}\text { Favia et al. (2018) } \\
\text { Pichardo et al. (2016) } \\
\text { Voss et al. (2012) } \\
\text { El-Rabbany et al. (2019) } \\
\text { Lopes et al. (2015) } \\
\text { Kim et al. (2017) } \\
\text { Hoefert et al. (2017) } \\
\text { Eguchi et al. (2017) } \\
\text { Ruggiero \& Kohn (2015) } \\
\text { Favia et al. (2016) }\end{array}$ & $\begin{array}{l}\text { Stockmann et al. (2010) } \\
\text { Rugani et al. (2015) } \\
\text { Hallmer et al. (2018) } \\
\text { Reich et al. (2015) }\end{array}$ & $\begin{array}{l}\text { Aljohani et al. (2018) } \\
\text { Pichardo \& van Merkesteyn } \\
\text { (2016) }\end{array}$ \\
\hline
\end{tabular}

Fonte: Autores.

Os medicamentos empregados nos tratamentos para ONMIM foram PENT-E (Owosho et al., 2016), Sitofloxacina (Ikeda et al., 2015) e Minociclina (Karasneh et al., 2016). Todos os autores consideraram as terapias de sucesso quanto à cicatrização das lesões.

Observou-se que o tratamento cirúrgico conservador (Lerman et al., 2013; Coropciuc et al., 2017; Nisi et al., 2018; Lee et al., 2014; Moretti et al., 2011; Fortuna et al., 2012; Thumbigere-Math et al., 2009) tem eficácia no tratamento da ONMIM. Cirurgias conservadoras apresentaram taxa de sucesso entre 79,73\% (Coropciuc et al., 2017) e 91,8\% (Nisi et al., 2018) após 24 meses de acompanhamento. Segundo os autores, o tratamento puramente conservador pode ser indicado levando em consideração os resultados incertos da terapia cirúrgica (Moretti et al., 2011), podendo alcançar melhora na sintomatologia dos pacientes (Moretti et al., 2011, Fortuna et al., 2012) e em estágios iniciais, pode até resolver completamente a lesão (Thumbigere-Math et al., 2009). 
As terapias teciduais (Mauceri et al., 2018; Inchingolo et al., 2017; Mourão et al., 2020; Calvani et al., 2018; Lemound et al., 2018; Coviello et al., 2012; Park et al., 2017; Jung et al., 2017; Aljohani et al., 2019; Mozzati et al., 2012; Curi et al., 2011; Rotaru et al., 2015) também demonstraram eficácia no tratamento das lesões de ONMIM. A utilização de retalhos de gordura bucal apresentou-se como uma modalidade de tratamento capaz de reduzir a duração da antibioticoterapia e de cobrir grandes defeitos cirúrgicos (Rotaru et al., 2015), principalmente em lesões caracterizadas em estágio 3 (Aljohani et al., 2019). A aplicação de Plasma Rico em Plaquetas autólogo (PRP) demonstrou eficácia na melhora da cicatrização (Mauceri et al., 2018; Coviello et al., 2012; Curi et al., 2011), bem como a aplicação de Plasma Rico em Fibrina (PRF) (Inchingolo et al., 2017; Mourão et al., 2020; Park et al., 2017), Plasma Rico em Fatores de Crescimento (PRGF) (Mozzati et al., 2012) e gazes embebidas com Lactoferrina Bovina (bLf) (Calvani et al., 2018). A utilização de retalhos nasolabiais (Lemound et al., 2018) demonstrou altas taxas de sucesso do grupo experimental. Os plugs de colágeno embebidos em rhBMP-2 (Jung et al., 2017) apresentaram benefícios durante a fase de cicatrização das lesões.

Verificaram-se sete modalidades de tratamento adjuvante para a ONMIM. Estes tratamentos foram: oxigenação hiperbárica (Bedogni et al., 2011; Freiberger et al., 2012), remoção óssea por laser Er: YAG (Atalay et al., 2011; Stübinger et al., 2009), ozonioterapia (Ripamonti et al., 2011; Agrillo et al., 2012), cirurgia guiada por autofluorescência (Giudice et al., 2018; Ristow et al., 2017), uso de Teriparatide (Kwon et al., 2012), fechamento assistido à vácuo (Laimer et al., 2017) e um estudo (Voss et al., 2017) apresentou o uso de células-tronco mesenquimais da crista ilíaca do paciente.

O tratamento à base de laser Er: YAG e a cirurgia guiada por autofluorescência, apesar de auxiliarem nos procedimentos cirúrgicos, como o efeito antimicrobiano do laser e a facilidade na delimitação das margens da lesão na cirurgia guiada, demonstraram resultados semelhantes à técnica convencional cirúrgica (Giudice et al., 2018; Ristow et al., 2017; Atalay et al., 2011). Já as técnicas envolvendo ozonioterapia, uso de Teriparatida, fechamento assistido a vácuo e o uso de células-tronco mesenquimais da crista ilíaca apresentaram benefícios para a fase de cicatrização da lesão.

O tratamento cirúrgico agressivo (Favia et al., 2018; Pichardo et al., 2016; Voss et al., 2012; El-Rabbany et al., 2019; Lopes et al., 2015; Kim et al., 2017; Hoefert et al., 2017; Eguchi et al., 2017; Ruggiero \& Kohn, 2015; Favia et al., 2016; Stockmann et al., 2010; Rugani et al., 2015; Hallmer et al., 2018; Reich et al., 2015; Aljohani et al., 2018; Pichardo \& van Merkesteyn, 2016), baseado principalmente na remoção total da lesão incluindo uma faixa de segurança de osso sadio,demonstrou eficácia para as lesões de ONMIM. As taxas de sucesso a partir deste tratamento variaram entre 70\% (ElRabbany et al., 2019; Kim et al., 2017) e 95,2\% (Voss et al., 2012), sendo que a taxa elevada foi alcançada por meio da associação entre remoção completa do osso afetado com o fechamento da ferida em três camadas. Os autores relataram que a remoção óssea até encontrar osso sangrante é um parâmetro de segurança confiável para definição das margens da lesão (Reich et al. 2015; Pichardo et al., 2016; Voss et al., 2012; El-Rabbany et al., 2019; Lopes et al., 2015; Kim et al., 2017; Pichardo \& van Merkesteyn, 2016; Mozzati et al., 2012). Quanto à relação entre a descontinuação do tratamento medicamentoso antirreabsortivo e a obtenção de melhores resultados de cicatrização, houve divergência entre publicações, sendo que alguns recomendam a descontinuação (Stockmann et al., 2010; Mourão et al., 2020; Eguchi et al., 2017) e outras relatam não haver diferenças estatísticas que justifiquem a descontinuação (Kim et al., 2017; Hoefert et al., 2017; Voss et al., 2017; Curi et al., 2011).

O tempo de acompanhamento dos pacientes após os procedimentos cirúrgicos agressivos variou entre 6 (Rugani et al., 2015; Pichardo et al., 2016; Kim et al., 2017; Eguchi et al., 2017; Ruggiero \& Kohn, 2015) e 24 (Voss et al., 2012) meses, não havendo relação com as taxas de sucesso encontradas nos tratamentos. 


\section{Discussão}

Os bisfosfonatos e o Denosumab são medicamentos antirreabsortivos capazes de suprimir a ação osteoclástica. Os bisfosfonatos, por sua afinidade com a hidroxiapatita, fixam-se ao osso e são absorvidos pelos osteoclastos no ato de reabsorção óssea. No interior da célula osteoclástica, os bisfosfonatos induzem a apoptose celular. Já o Denosumab atua como anti-receptor ativador monoclonal do fator nuclear Kappa B ligante ou RANKL, inibindo a ligação entre RANK e RANKL e, assim impedindo a sobrevivência dos osteoclastos. (Roelofs, Thompson, Ebetino, Rogers \& Coxon, 2010; Baron, Ferrari \& Russell, 2011). Os agentes anti-fator de crescimento endotelial vascular (VEGF) apresentam atividade antiangiogênica. Estes medicamentos apresentam importância fundamental na prevenção das metástases relacionadas aos cânceres, além de privar massas tumorais de suprimento sanguíneo, prevenindo ou retardando o crescimento do tumor (Folkman, 2003; El-Kenawi \& El-Remessy, 2013).

Segundo estudos publicados, a Osteonecrose dos Maxilares Associada ao uso de Medicamentos ocorre mais frequentemente em idosos e diante de administração intravenosa, principalmente do Zolendronato (Khan et al., 2015; Fliefel, Tröltzsch, Kühnisch, Ehrenfeld \& Otto, 2015). Estas informações foram confirmadas com este estudo, visto que a média de idade encontrada foi de 63,06 anos, e os pacientes portadores de câncer estavam sob tratamento do Zolendronato por via intravenosa.

Ruggiero et al. (2014) relatou que a ONMIM acomete principalmente a mandíbula. Segundo Saad et al. (2012), a mandíbula isoladamente é acometida em $73 \%$ dos casos, a maxila em $22,5 \%$ dos casos e ambos os maxilares são acometidos em $4,5 \%$ dos casos. Este estudo corroborou as informações quanto à localização das lesões, visto que $68,8 \%$ dos pacientes apresentaram lesões mandibulares, $27,4 \%$ dos pacientes demonstraram lesões maxilares e apenas $3,7 \%$ tiveram ambos os maxilares acometidos.

Diferente da declaração da Food and Drug Administration of United States que concluiu, no ano de 2011, que a suspensão dos medicamentos não era necessária antes das cirurgias (Hellstein et al., 2011), três publicações incluídas nesta revisão recomendaram a descontinuação do medicamento (Stockmann et al., 2010; Mourão et al., 2020; Eguchi et al., 2017). Estes estudos demonstraram taxas de sucesso entre $89 \%$ e $100 \%$, porém todos os três estudos apresentaram pacientes sob o uso oral de bifosfonato para tratamento de osteoporose. Talvez a meia vida menor dos medicamentos administrados por via oral, de modo que a suspensão diminua a concentração óssea dos medicamentos, tenha influencia na indicação.

Todos os medicamentos relatados apresentaram-se benéficos ao tratamento. A PENT-E (Owosho et al., 2016) foi capaz de aliviar o sintoma em todos os sete pacientes, a Sitofloxacina (Ikeda et al., 2015) foi capaz de controlar ou curar completamente a doença em 95\% dos casos e a Minociclina (Karasneh et al., 2016) promoveu a cura completa em 60\% dos pacientes. Todos os pacientes tratados com Minociclina eram portadores de diabetes, o que pode ter influenciado na menor taxa de sucesso dentre os três medicamentos.

Esta revisão englobou sete estudos associados a tratamentos cirúrgicos conservadores para a ONMIM, e observou-se certa eficácia relacionada a esta modalidade de tratamento. Nisi et al. (2018) demonstraram que o debridamento supragengival associado ao uso de enxaguatórios bucais com clorexidina a 0,2\% e Amoxicilina com Ácido Clavulânico por 14 dias foi capaz de estabilizar a ONMIM e aliviar a dor nos pacientes, porém a cura só foi alcançada após tratamento cirúrgico conservador, envolvendo sequestrectomia e curetagem óssea limitada. Esta conclusão se assemelha com a alcançada por outros autores (Lee et al., 2014; Fortuna et al., 2012). Apesar da alta taxa de encontrada por Nisi et al. (2018) é válido pontuar que todos os pacientes do estudo eram portadores de osteoporose, e utilizavam medicamento por via oral. Talvez a maior taxa de sucesso possa estar associada ao tratamento e à doença previamente tratada. 
Outros estudos (Thumbigere-Math et al., 2009; Moretti et al., 2011; Lerman et al., 2013; Coropciuc et al., 2017) também demonstraram a eficácia da cirurgia conservadora, chegando a conclusões como: lesões caracterizadas como estágio 1 apresentam maior chance de cura por esta técnica (Coropciuc et al., 2017), lesões espontâneas respondem melhor em comparação a lesões que se desenvolveram após procedimento dentoalveolar (Thumbigere-Math et al., 2009), cirurgias mais extensas devem ser reservadas para lesões em estágio 3 e/ou lesões refratárias a tratamentos anteriores (Lerman et al., 2013) e que o grande custo biológico e a imprevisibilidade são justificativas para evitar cirurgias agressivas, tendo em vista que os pacientes normalmente já se apresentam comprometidos (Moretti et al., 2011).

As terapias teciduais (Mauceri et al., 2018; Inchingolo et al., 2017; Mourão et al., 2020; Calvani et al., 2018; Lemound et al., 2018; Coviello et al., 2012; Park et al., 2017; Jung et al., 2017; Aljohani et al., 2019; Mozzati et al., 2012; Curi et al., 2011; Rotaru et al., 2015) demonstraram serem benéficas para o tratamento do paciente. O uso do retalho de gordura bucal (Rotaru et al., 2015; Aljohani et al., 2019) se mostrou superior ao fechamento mucoperiosteal das feridas, com taxas de cicatrização completa do tecido mole entre 85,7\% (Aljohani et al., 2019) e 100\% (Rotaru et al., 2015), apesar do estudo que apresentou tal eficácia em todos os pacientes ter englobado apenas 10 pacientes. Os retalhos de gordura bucal permitiram o fechamento confiável das feridas de até $62 \mathrm{~mm}$ x $18 \mathrm{~mm}$, principalmente em lesões classificadas como estágio 3 . A aplicação de PRP autólogo também demonstrou ser benéfico no tratamento, alcançado taxas de sucesso em $80 \%$ dos casos (Mauceri et al., 2018; Coviello et al., 2012; Curi et al., 2011).

A Plaqueta Rica em Fibrina (PRF) apresentou elevada taxa de sucesso. Um total de 34 pacientes foi tratado com uso de PRF (Inchingolo et al., 2017; Mourão et al., 2020) e todos apresentaram resultados bem sucedidos, com cicatrização completa dos tecidos moles. Apesar do excelente resultado, 23 pacientes só foram acompanhados por 30 dias, e o estágio das lesões destes não foi informada (Inchingolo et al., 2017). A associação entre L-PRF e BMP-2 (Park et al., 2017) demonstrou ser superior ao uso isolado do L-PRF, com taxas de sucesso de $60 \%$ e $36 \%$ respectivamente. A preparação enriquecida com plaquetas (PRGF) apresentou taxa de 100\% de sucesso (Mozzati et al., 2012), entretanto, o acompanhamento destes pacientes foi deficiente, visto que após 60 meses, apenas 17 dos 32 pacientes apresentaram retorno.

Os retalhos nasolabiais (Lemound et al., 2018) e o uso de gazes embebidas em bLf (Calvani et al., 2018) também demonstraram ser superiores ao protocolo convencional, consistindo apenas na cirurgia e fechamento com suturas mucoperiosteais, sendo que o primeiro apresentou cicatrização de $68,8 \%$ das lesões e o segundo de $100 \%$ das lesões. A terapia auxiliada com gazes embebidas em bLf apresentou destaque, pois mesmo em um período de acompanhamento de seis meses, as 13 lesões classificadas como estágio 2 permaneceram cicatrizadas, porém mais estudos sobre a técnica precisam ser feitos para comprovar a real eficácia. O uso de rhBMP-2 associada à Teriparatida apresentou-se superior se comparado com o grupo controle, diferentemente do uso isolado da rhBMP-2, que não apresentou diferença estatística com o grupo controle (Jung et al., 2017).

Sobre as terapias adjuvantes, a taxa de sucesso da terapia com oxigenação hiperbárica teve um declínio de 90,6\% em um tempo de acompanhamento de até 6 meses (Bedogni et al., 2011) para 68\% em um tempo de acompanhamento de até 18 meses (Freiberger et al., 2012). O tempo de acompanhamento pode ser o fator que justifica esta diferença nas taxas. Um período maior de acompanhamento pode demonstrar insucessos que acompanhamento em curto prazo não foi capaz de demonstrar. Com o passar do tempo, o paciente pode perder a motivação em cuidar da lesão e se não houver descontinuação do tratamento anti-resorptivo ou antiangiogênico podem ainda surgir novas lesões ou a lesão tratada pode recidivar.

A remoção óssea com laser Er. YAG e as cirurgias guiadas por autofluorescência, apesar de demonstrarem eficácia no tratamento da ONMIM, não apresentaram resultados superiores se comparado com a técnica convencional baseada no debridamento da lesão. Diferentemente do uso da Teriparatida (Kwon et al., 2012) que demonstrou cura completa de todos os 
pacientes, apesar de que o tempo de avaliação foi curto, sendo de três meses. O uso de células-tronco mesenquimais da crista ilíaca (Voss et al., 2017) apresentou excelente resultado no tratamento, com 83,3\% de taxa de sucesso em um período de acompanhamento de 18 meses em pacientes estágio 1 e 2 . A ozonioterapia foi superior aos tratamentos convencionais, com índice de cura completa variando entre 60,6\%, com média de 4 sessões (Agrillo et al., 2012), e 70\% com até 7 sessões (Ripamonti et al., 2011). Um relato publicado recentemente demonstrou que a ozonioterapia pode ser importante para casos de ONMIM em pacientes, que por alguma contraindicação médica, são impossibilitados de receber cirurgias. Tendo a capacidade, inclusive, de levar a esfoliação do sequestro ósseo, permitindo assim o recobrimento epitelial da lesão (Lima, Paulo, Silva, Filice \& Barbosa, 2020).

O tratamento cirúrgico agressivo (Favia et al., 2018; Pichardo et al., 2016; Voss et al., 2012; El-Rabbany et al., 2019; Lopes et al., 2015; Kim et al., 2017; Hoefert et al., 2017; Eguchi et al., 2017; Ruggiero \& Kohn, 2015; Favia et al., 2016; Stockmann et al., 2010; Rugani et al., 2015; Hallmer et al., 2018; Reich et al., 2015; Aljohani et al., 2018; Pichardo \& van Merkesteyn, 2016) foi o que apresentou melhores resultados no tratamento da ONMIM. A taxa mais alta encontrada foi relacionada com a remoção completa do osso até atingir osso sangrante em feridas estágio 2 e 3 , com fechamento da ferida em três camadas e interrupção do tratamento antirreabsortivo 4 semanas antes e 6 semanas depois da cirurgia (Voss et al., 2012). Este estudo mostrou que o fechamento confiável dos tecidos moles permite a obtenção de resultados melhores e mais previsíveis. Esta técnica foi capaz de resultar em melhora de 95,2\% dos pacientes, com período de acompanhamento de 24 meses. Em contrapartida, a menor taxa de sucesso encontrada foi de 70\% (Kim et al., 2017). Este resultado encontrado pode estar associado à maior coorte de estudo, com 325 pacientes e às lesões majoritariamente classificadas como estágio 3.

Por fim, apesar das várias terapias empregadas para o tratamento da Osteonecrose dos Maxilares Associada ao uso de Medicamentos, ainda não há um consenso sobre o melhor tratamento. Terapias medicamentosas demonstraram bons resultados, porém poucos estudos estão presentes na literatura sobre este tema. As terapias teciduais, com uso principalmente de PRF e PRP, têm sido vastamente estudadas e demonstram excelentes resultados, podendo ser uma opção interessante para o arsenal de tratamento do cirurgião. O mesmo pode ser dito para a oxigenação hiperbárica. A literatura apresenta muitos relatos sobre osteonecroses tratadas com cirurgias agressivas, pois esta terapia já provou resultar em alta taxa de sucesso, particularmente para lesões em estágios mais avançados. Porém, deve-se lembrar que toda cirurgia agressiva é acompanhada de uma série de consequências e estas podem não ser bem toleradas por pacientes, principalmente idosos. Neste cenário, as cirurgias conservadoras apresentam importância. Apesar de nem sempre conseguirem a cura completa da lesão, a remissão dos sintomas frequentemente é alcançada, devolvendo qualidade de vida ao paciente.

\section{Considerações Finais}

Apesar da baixa incidência, as lesões de osteonecrose dos maxilares associada ao uso de medicamentos são extremamente desconfortáveis para os pacientes. Diante disso, o cirurgião dentista deve estar apto a diagnosticar e tratar estas lesões. Vários tratamentos são propostos, seja medicamentoso, cirúrgico agressivo, cirúrgico conservador, associado a terapias teciduais ou a partir de técnicas adjuvantes. Parece claro que as cirurgias agressivas promovem melhores resultados, principalmente se associados a uma terapia extra, seja tecidual ou adjuvante. No entanto, o cirurgião-dentista deve sempre ter em mente a condição de saúde do paciente e sua possível influencia no prognóstico do tratamento a ser escolhido.

Mais estudos quanto ao tratamento da ONMIM devem ser incentivados, tendo em vista que a expectativa de vida tem aumentado, e assim, mais frequente será a presença de pacientes sob o uso de medicamentos relacionados a esta condição no consultório odontológico. 


\section{Referências}

Advisory Task Force on Bisphosphonate-Related Ostenonecrosis of the Jaws, American Association of Oral and Maxillofacial Surgeons (2007). American Association of Oral and Maxillofacial Surgeons position paper on bisphosphonate-related osteonecrosis of the jaws. Journal of oral and maxillofacial surgery: official journal of the American Association of Oral and Maxillofacial Surgeons, 65(3), 369-376.

Agrillo, A., Filiaci, F., Ramieri, V., Riccardi, E., Quarato, D., Rinna, C., Gennaro, P., Cascino, F., Mitro, V., \& Ungari, C. (2012). Bisphosphonate-related osteonecrosis of the jaw (BRONJ): 5-year experience in the treatment of 131 cases with ozone therapy. European review for medical and pharmacological sciences, 16(12), 1741-1747.

Aljohani, S., Gaudin, R., Weiser, J., Tröltzsch, M., Ehrenfeld, M., Kaeppler, G., Smeets, R., \& Otto, S. (2018). Osteonecrosis of the jaw in patients treated with denosumab: A multicenter case series. Journal of cranio-maxillo-facial surgery: official publication of the European Association for Cranio-MaxilloFacial Surgery, 46(9), 1515-1525.

Aljohani, S., Troeltzsch, M., Hafner, S., Kaeppler, G., Mast, G., \& Otto, S. (2019). Surgical treatment of medication-related osteonecrosis of the upper jaw: Case series. Oral diseases, 25(2), 497-507.

Atalay, B., Yalcin, S., Emes, Y., Aktas, I., Aybar, B., Issever, H., Mandel, N. M., Cetin, O., \& Oncu, B. (2011). Bisphosphonate-related osteonecrosis: laserassisted surgical treatment or conventional surgery?. Lasers in medical science, 26(6), 815-823.

Baron, R., Ferrari, S., \& Russell, R. G. (2011). Denosumab and bisphosphonates: different mechanisms of action and effects. Bone, 48(4), 677-692.

Bedogni, A., Saia, G., Bettini, G., Tronchet, A., Totola, A., Bedogni, G., Ferronato, G., Nocini, P. F., \& Blandamura, S. (2011). Long-term outcomes of surgical resection of the jaws in cancer patients with bisphosphonate-related osteonecrosis. Oral oncology, 47(5), $420-424$.

Calvani, F., Cutone, A., Lepanto, M. S., Rosa, L., Valentini, V., \& Valenti, P. (2018). Efficacy of bovine lactoferrin in the post-surgical treatment of patients suffering from bisphosphonate-related osteonecrosis of the jaws: an open-label study. Biometals: an international journal on the role of metal ions in biology, biochemistry, and medicine, 31(3), 445-455.

Coropciuc, R. G., Grisar, K., Aerden, T., Schol, M., Schoenaers, J., \& Politis, C. (2017). Medication-related osteonecrosis of the jaw in oncological patients with skeletal metastases: conservative treatment is effective up to stage 2. The British journal of oral \& maxillofacial surgery, 55(8), 787-792.

Coviello, V., Peluso, F., Dehkhargani, S. Z., Verdugo, F., Raffaelli, L., Manicone, P. F., \& D' Addona, A. (2012). Platelet-rich plasma improves wound healing in multiple myeloma bisphosphonate-associated osteonecrosis of the jaw patients. Journal of biological regulators and homeostatic agents, 26(1), $151-155$.

Curi, M. M., Cossolin, G. S., Koga, D. H., Zardetto, C., Christianini, S., Feher, O., Cardoso, C. L., \& dos Santos, M. O. (2011). Bisphosphonate-related osteonecrosis of the jaws--an initial case series report of treatment combining partial bone resection and autologous platelet-rich plasma. Journal of oral and maxillofacial surgery: official journal of the American Association of Oral and Maxillofacial Surgeons, 69(9), 2465-2472.

Eguchi, T., Kanai, I., Basugi, A., Miyata, Y., Inoue, M., \& Hamada, Y. (2017). The assessment of surgical and non-surgical treatment of stage II medicationrelated osteonecrosis of the jaw. Medicina oral, patologia oral y cirugia bucal, 22(6), e788-e795.

El-Kenawi, A. E., \& El-Remessy, A. B. (2013). Angiogenesis inhibitors in cancer therapy: mechanistic perspective on classification and treatment rationales. British journal of pharmacology, 170(4), 712-729.

El-Rabbany, M., Lam, D. K., Shah, P. S., \& Azarpazhooh, A. (2019). Surgical Management of Medication-Related Osteonecrosis of the Jaw Is Associated With Improved Disease Resolution: A Retrospective Cohort Study. Journal of oral and maxillofacial surgery: official journal of the American Association of Oral and Maxillofacial Surgeons, 77(9), 1816-1822.

Favia, G., Tempesta, A., Limongelli, L., Crincoli, V., \& Maiorano, E. (2016). Medication-Related Osteonecrosis of the Jaws: Considerations on a New Antiresorptive Therapy (Denosumab) and Treatment Outcome after a 13-Year Experience. International journal of dentistry, $2016,1801676$.

Favia, G., Tempesta, A., Limongelli, L., Crincoli, V., \& Maiorano, E. (2018). Medication-related osteonecrosis of the jaw: Surgical or non-surgical treatment?. Oral diseases, 24(1-2), 238-242.

Mourão, C.F.A.B., Calasans-Maia, M. D., Del Fabbro, M., Le Drapper Vieira, F., Coutinho de Mello Machado, R., Capella, R., Miron, R. J., \& Gomes Alves, G. (2020). The use of Platelet-rich Fibrin in the management of medication-related osteonecrosis of the jaw: A case series. Journal of stomatology, oral and maxillofacial surgery, 121(1), 84-89.

Fizazi, K., Carducci, M., Smith, M., Damião, R., Brown, J., Karsh, L., Milecki, P., Shore, N., Rader, M., Wang, H., Jiang, Q., Tadros, S., Dansey, R., \& Goessl, C. (2011). Denosumab versus zoledronic acid for treatment of bone metastases in men with castration-resistant prostate cancer: a randomised, doubleblind study. Lancet (London, England), 377(9768), 813-822.

Fliefel, R., Tröltzsch, M., Kühnisch, J., Ehrenfeld, M., \& Otto, S. (2015). Treatment strategies and outcomes of bisphosphonate-related osteonecrosis of the jaw (BRONJ) with characterization of patients: a systematic review. International journal of oral and maxillofacial surgery, 44(5), 568-585.

Folkman J. (2003). Angiogenesis inhibitors: a new class of drugs. Cancer biology \& therapy, 2(4 Suppl 1), S127-S133.

Fortuna, G., Ruoppo, E., Pollio, A., Aria, M., Adamo, D., Leuci, S., Orabona, G. D., \& Mignogna, M. D. (2012). Multiple myeloma vs. breast cancer patients with bisphosphonates-related osteonecrosis of the jaws: a comparative analysis of response to treatment and predictors of outcome. Journal of oral pathology \& medicine: official publication of the International Association of Oral Pathologists and the American Academy of Oral Pathology, 41(3), 222-228.

Freiberger, J. J., Padilla-Burgos, R., McGraw, T., Suliman, H. B., Kraft, K. H., Stolp, B. W., Moon, R. E., \& Piantadosi, C. A. (2012). What is the role of hyperbaric oxygen in the management of bisphosphonate-related osteonecrosis of the jaw: a randomized controlled trial of hyperbaric oxygen as an adjunct to 
surgery and antibiotics. Journal of oral and maxillofacial surgery: official journal of the American Association of Oral and Maxillofacial Surgeons, 70(7), $1573-1583$

Giudice, A., Bennardo, F., Barone, S., Antonelli, A., Figliuzzi, M. M., \& Fortunato, L. (2018). Can Autofluorescence Guide Surgeons in the Treatment of Medication-Related Osteonecrosis of the Jaw? A Prospective Feasibility Study. Journal of oral and maxillofacial surgery: official journal of the American Association of Oral and Maxillofacial Surgeons, 76(5), 982-995.

Goldvaser, H., \& Amir, E. (2019). Role of Bisphosphonates in Breast Cancer Therapy. Current treatment options in oncology, $20(4), 26$.

Guarneri, V., Miles, D., Robert, N., Diéras, V., Glaspy, J., Smith, I., Thomssen, C., Biganzoli, L., Taran, T., \& Conte, P. (2010). Bevacizumab and osteonecrosis of the jaw: incidence and association with bisphosphonate therapy in three large prospective trials in advanced breast cancer. Breast cancer research and treatment, 122(1), 181-188.

Hallmer, F., Andersson, G., Götrick, B., Warfvinge, G., Anderud, J., \& Bjørnland, T. (2018). Prevalence, initiating factor, and treatment outcome of medication-related osteonecrosis of the jaw-a 4-year prospective study. Oral surgery, oral medicine, oral pathology and oral radiology, $126(6), 477-485$.

Hellstein, J. W., Adler, R. A., Edwards, B., Jacobsen, P. L., Kalmar, J. R., Koka, S., Migliorati, C. A., Ristic, H., \& American Dental Association Council on Scientific Affairs Expert Panel on Antiresorptive Agents (2011). Managing the care of patients receiving antiresorptive therapy for prevention and treatment of osteoporosis: executive summary of recommendations from the American Dental Association Council on Scientific Affairs. Journal of the American Dental Association (1939), 142(11), 1243-1251.

Hoefert, S., Yuan, A., Munz, A., Grimm, M., Elayouti, A., \& Reinert, S. (2017). Clinical course and therapeutic outcomes of operatively and non-operatively managed patients with denosumab-related osteonecrosis of the jaw (DRONJ). Journal of cranio-maxillo-facial surgery: official publication of the European Association for Cranio-Maxillo-Facial Surgery, 45(4), 570-578.

Ikeda, T., Kuraguchi, J., Kogashiwa, Y., Yokoi, H., Satomi, T., \& Kohno, N. (2015). Successful treatment of bisphosphonate-related osteonecrosis of the jaw (BRONJ) patients with sitafloxacin: new strategies for the treatment of BRONJ. Bone, 73, 217-222.

Inchingolo, F., Cantore, S., Dipalma, G., Georgakopoulos, I., Almasri, M., Gheno, E., Motta, A., Marrelli, M., Farronato, D., Ballini, A., \& Marzullo, A. (2017). Platelet rich fibrin in the management of medication-related osteonecrosis of the jaw: a clinical and histopathological evaluation. Journal of biological regulators and homeostatic agents, 31(3), 811-816.

Jung, J., Yoo, H. Y., Kim, G. T., Lee, J. W., Lee, Y. A., Kim, D. Y., \& Kwon, Y. D. (2017). Short-Term Teriparatide and Recombinant Human Bone Morphogenetic Protein-2 for Regenerative Approach to Medication-Related Osteonecrosis of the Jaw: A Preliminary Study. Journal of bone and mineral research : the official journal of the American Society for Bone and Mineral Research, 32(12), 2445-2452.

Karasneh, J. A., Al-Eryani, K., Clark, G. T., \& Sedghizadeh, P. P. (2016). Modified protocol including topical minocycline in orabase to manage medicationrelated osteonecrosis of the jaw cases. Journal of oral pathology \& medicine: official publication of the International Association of Oral Pathologists and the American Academy of Oral Pathology, 45(9), 718-720.

Khan A.A., Morrison A., Hanley D.A., Felsenberg D., McCauley L.K., O'Ryan F., Reid I.R., Ruggiero S.L., Taguchi A., Tetradis S., Watts N.B., Brandi M.L., Peters E., Guise T., Eastell R., Cheung A.M., Morin S.N., Masri B., Cooper C., Morgan S.L., Obermayer-Pietsch B., Langdahl B.L., Al Dabagh R., Davison K.S., Kendler D.L., Sándor G.K., Josse R.G., Bhandari M., El Rabbany M., Pierroz D.D., Sulimani R., Saunders D.P., Brown J.P., Compston J. \& International Task Force on Osteonecrosis of the Jaw (2015). Diagnosis and management of osteonecrosis of the jaw: a systematic review and international consensus. Journal of bone and mineral research: the official journal of the American Society for Bone and Mineral Research, 30(1), 3-23.

Kim, H. Y., Lee, S. J., Kim, S. M., Myoung, H., Hwang, S. J., Choi, J. Y., Lee, J. H., Choung, P. H., Kim, M. J., \& Seo, B. M. (2017). Extensive Surgical Procedures Result in Better Treatment Outcomes for Bisphosphonate-Related Osteonecrosis of the Jaw in Patients With Osteoporosis. Journal of oral and maxillofacial surgery: official journal of the American Association of Oral and Maxillofacial Surgeons, 75(7), 1404-1413.

Kwon, Y. D., Lee, D. W., Choi, B. J., Lee, J. W., \& Kim, D. Y. (2012). Short-term teriparatide therapy as an adjunctive modality for bisphosphonate-related osteonecrosis of the jaws. Osteoporosis international : a journal established as result of cooperation between the European Foundation for Osteoporosis and the National Osteoporosis Foundation of the USA, 23(11), 2721-2725.

Laimer, J., Steinmassl, O., Hechenberger, M., Rasse, M., Pikula, R., \& Bruckmoser, E. (2017). Intraoral Vacuum-Assisted Closure Therapy-A Pilot Study in Medication-Related Osteonecrosis of the Jaw. Journal of oral and maxillofacial surgery: official journal of the American Association of Oral and Maxillofacial Surgeons, 75(10), 2154-2161.

Lee, L. W., Hsiao, S. H., \& Chen, L. K. (2014). Clinical treatment outcomes for 40 patients with bisphosphonates-related osteonecrosis of the jaws. Journal of the Formosan Medical Association = Taiwan yi zhi, 113(3), 166-172.

Lemound, J., Muecke, T., Zeller, A. N., Lichtenstein, J., Eckardt, A., \& Gellrich, N. C. (2018). Nasolabial Flap Improves Healing in Medication-Related Osteonecrosis of the Jaw. Journal of oral and maxillofacial surgery: official journal of the American Association of Oral and Maxillofacial Surgeons, 76(4), $877-885$.

Lerman, M. A., Xie, W., Treister, N. S., Richardson, P. G., Weller, E. A., \& Woo, S. B. (2013). Conservative management of bisphosphonate-related osteonecrosis of the jaws: staging and treatment outcomes. Oral oncology, 49(9), 977-983.

Lima, L. B., Paulo, L. F. B., Silva, C. J., Filice, L. de S. C., Barbosa, G. L. R. (2020). Osteonecrose dos maxilares associado ao uso de medicamentos (OMAM) em estágio 3: tratamento conservador bem sucedido com ozonioterapia. Research, Society and Development, [S. l.], 9(12), e44591211212.

Lopes, R. N., Rabelo, G. D., Rocha, A. C., Carvalho, P. A., \& Alves, F. A. (2015). Surgical Therapy for Bisphosphonate-Related Osteonecrosis of the Jaw: Six-Year Experience of a Single Institution. Journal of oral and maxillofacial surgery: official journal of the American Association of Oral and Maxillofacial Surgeons, 73(7), 1288-1295. 
Mauceri, R., Panzarella, V., Maniscalco, L., Bedogni, A., Licata, M. E., Albanese, A., Toia, F., Cumbo, E., Mazzola, G., Di Fede, O., \& Campisi, G. (2018). Conservative Surgical Treatment of Bisphosphonate-Related Osteonecrosis of the Jaw with Er,Cr:YSGG Laser and Platelet-Rich Plasma: A Longitudinal Study. BioMed research international, 2018, 3982540.

Moretti, F., Pelliccioni, G. A., Montebugnoli, L., \& Marchetti, C. (2011). A prospective clinical trial for assessing the efficacy of a minimally invasive protocol in patients with bisphosphonate-associated osteonecrosis of the jaws. Oral surgery, oral medicine, oral pathology, oral radiology, and endodontics, 112(6), 777-782.

Mozzati, M., Gallesio, G., Arata, V., Pol, R., \& Scoletta, M. (2012). Platelet-rich therapies in the treatment of intravenous bisphosphonate-related osteonecrosis of the jaw: a report of 32 cases. Oral oncology, 48(5), 469-474.

Nisi, M., Karapetsa, D., Gennai, S., Ramaglia, L., Graziani, F., \& Gabriele, M. (2018). Conservative surgical treatment of medication related osteonecrosis of the jaw (MRONJ) lesions in patients affected by osteoporosis exposed to oral bisphosphonates: 24 months follow-up. Journal of cranio-maxillo-facial surgery: official publication of the European Association for Cranio-Maxillo-Facial Surgery, 46(7), 1153-1158.

Owosho, A. A., Estilo, C. L., Huryn, J. M., \& Yom, S. K. (2016). Pentoxifylline and tocopherol in the management of cancer patients with medication-related osteonecrosis of the jaw: an observational retrospective study of initial case series. Oral surgery, oral medicine, oral pathology and oral radiology, 122(4), $455-459$.

Papapoulos, S., Chapurlat, R., Libanati, C., Brandi, M. L., Brown, J. P., Czerwiński, E., Krieg, M. A., Man, Z., Mellström, D., Radominski, S. C., Reginster, J. Y., Resch, H., Román Ivorra, J. A., Roux, C., Vittinghoff, E., Austin, M., Daizadeh, N., Bradley, M. N., Grauer, A., Cummings, S. R., \& Bone, H. G. (2012). Five years of denosumab exposure in women with postmenopausal osteoporosis: results from the first two years of the FREEDOM extension. Journal of bone and mineral research: the official journal of the American Society for Bone and Mineral Research, 27(3), 694-701.

Park, J. H., Kim, J. W., \& Kim, S. J. (2017). Does the Addition of Bone Morphogenetic Protein 2 to Platelet-Rich Fibrin Improve Healing After Treatment for Medication-Related Osteonecrosis of the Jaw?. Journal of oral and maxillofacial surgery: official journal of the American Association of Oral and Maxillofacial Surgeons, 75(6), 1176-1184.

Pereira, A.S., Shitsuka, D.M., Parreira, F.J. \& Shitsuka, R. (2018). Metodologia em pesquisa científica. UFSM - Santa Maria, RS. 1

Pichardo, S. E., \& van Merkesteyn, J. P. (2016). Evaluation of a surgical treatment of denosumab-related osteonecrosis of the jaws. Oral surgery, oral medicine, oral pathology and oral radiology, 122(3), 272-278.

Pichardo, S. E., Kuijpers, S. C., \& van Merkesteyn, J. P. (2016). Bisphosphonate-related osteonecrosis of the jaws: Cohort study of surgical treatment results in seventy-four stage II/III patients. Journal of cranio-maxillo-facial surgery: official publication of the European Association for Cranio-Maxillo-Facial Surgery, 44(9), 1216-1220.

Qi, W. X., Tang, L. N., He, A. N., Yao, Y., \& Shen, Z. (2014). Risk of osteonecrosis of the jaw in cancer patients receiving denosumab: a meta-analysis of seven randomized controlled trials. International journal of clinical oncology, 19(2), 403-410.

Reich, W., Bilkenroth, U., Schubert, J., Wickenhauser, C., \& Eckert, A. W. (2015). Surgical treatment of bisphosphonate-associated osteonecrosis: Prognostic score and long-term results. Journal of cranio-maxillo-facial surgery: official publication of the European Association for Cranio-Maxillo-Facial Surgery, 43(9), 1809-1822.

Ripamonti, C. I., Cislaghi, E., Mariani, L., \& Maniezzo, M. (2011). Efficacy and safety of medical ozone (O(3)) delivered in oil suspension applications for the treatment of osteonecrosis of the jaw in patients with bone metastases treated with bisphosphonates: Preliminary results of a phase I-II study. Oral oncology, 47(3), 185-190.

Ristow, O., Otto, S., Geiß, C., Kehl, V., Berger, M., Troeltzsch, M., Koerdt, S., Hohlweg-Majert, B., Freudlsperger, C., \& Pautke, C. (2017). Comparison of auto-fluorescence and tetracycline fluorescence for guided bone surgery of medication-related osteonecrosis of the jaw: a randomized controlled feasibility study. International journal of oral and maxillofacial surgery, 46(2), 157-166.

Rodan, G. A., \& Fleisch, H. A. (1996). Bisphosphonates: mechanisms of action. The Journal of clinical investigation, 97(12), 2692-2696.

Roelofs, A. J., Thompson, K., Ebetino, F. H., Rogers, M. J., \& Coxon, F. P. (2010). Bisphosphonates: molecular mechanisms of action and effects on bone cells, monocytes and macrophages. Current pharmaceutical design, 16(27), 2950-2960.

Rotaru, H., Kim, M. K., Kim, S. G., \& Park, Y. W. (2015). Pedicled buccal fat pad flap as a reliable surgical strategy for the treatment of medication-related osteonecrosis of the jaw. Journal of oral and maxillofacial surgery: official journal of the American Association of Oral and Maxillofacial Surgeons, 73(3), $437-442$.

Rugani, P., Acham, S., Kirnbauer, B., Truschnegg, A., Obermayer-Pietsch, B., \& Jakse, N. (2015). Stage-related treatment concept of medication-related osteonecrosis of the jaw-a case series. Clinical oral investigations, 19(6), 1329-1338.

Ruggiero, S. L., \& Kohn, N. (2015). Disease Stage and Mode of Therapy Are Important Determinants of Treatment Outcomes for Medication-Related Osteonecrosis of the Jaw. Journal of oral and maxillofacial surgery: official journal of the American Association of Oral and Maxillofacial Surgeons, 73(12 Suppl), S94-S100.

Ruggiero, S. L., Dodson, T. B., Assael, L. A., Landesberg, R., Marx, R. E., Mehrotra, B., \& Task Force on Bisphosphonate-Related Osteonecrosis of the Jaws, American Association of Oral and Maxillofacial Surgeons (2009). American Association of Oral and Maxillofacial Surgeons position paper on bisphosphonate-related osteonecrosis of the jaw - 2009 update. Australian endodontic journal: the journal of the Australian Society of Endodontology Inc, 35(3), 119-130.

Ruggiero, S. L., Dodson, T. B., Fantasia, J., Goodday, R., Aghaloo, T., Mehrotra, B., O'Ryan, F., \& American Association of Oral and Maxillofacial Surgeons (2014). American Association of Oral and Maxillofacial Surgeons position paper on medication-related osteonecrosis of the jaw--2014 update. Journal of oral and maxillofacial surgery: official journal of the American Association of Oral and Maxillofacial Surgeons, 72(10), 1938-1956. 
Research, Society and Development, v. 10, n. 2, e4210212168, 2021

(CC BY 4.0) | ISSN 2525-3409 | DOI: http://dx.doi.org/10.33448/rsd-v10i2.12168

Saad, F., Brown, J. E., Van Poznak, C., Ibrahim, T., Stemmer, S. M., Stopeck, A. T., Diel, I. J., Takahashi, S., Shore, N., Henry, D. H., Barrios, C. H., Facon, T., Senecal, F., Fizazi, K., Zhou, L., Daniels, A., Carrière, P., \& Dansey, R. (2012). Incidence, risk factors, and outcomes of osteonecrosis of the jaw: integrated analysis from three blinded active-controlled phase III trials in cancer patients with bone metastases. Annals of oncology: official journal of the European Society for Medical Oncology, 23(5), 1341-1347.

Stockmann, P., Vairaktaris, E., Wehrhan, F., Seiss, M., Schwarz, S., Spriewald, B., Neukam, F. W., \& Nkenke, E. (2010). Osteotomy and primary wound closure in bisphosphonate-associated osteonecrosis of the jaw: a prospective clinical study with 12 months follow-up. Supportive care in cancer: official journal of the Multinational Association of Supportive Care in Cancer, 18(4), 449-460.

Stübinger, S., Dissmann, J. P., Pinho, N. C., Saldamli, B., Seitz, O., \& Sader, R. (2009). A preliminary report about treatment of bisphosphonate related osteonecrosis of the jaw with Er:YAG laser ablation. Lasers in surgery and medicine, 41(1), 26-30.

Thumbigere-Math, V., Sabino, M. C., Gopalakrishnan, R., Huckabay, S., Dudek, A. Z., Basu, S., Hughes, P. J., Michalowicz, B. S., Leach, J. W., Swenson, K. K., Swift, J. Q., Adkinson, C., \& Basi, D. L. (2009). Bisphosphonate-related osteonecrosis of the jaw: clinical features, risk factors, management, and treatment outcomes of 26 patients. Journal of oral and maxillofacial surgery: official journal of the American Association of Oral and Maxillofacial Surgeons, 67(9), 1904-1913.

Voss, P. J., Joshi Oshero, J., Kovalova-Müller, A., Veigel Merino, E. A., Sauerbier, S., Al-Jamali, J., Lemound, J., Metzger, M. C., \& Schmelzeisen, R. (2012). Surgical treatment of bisphosphonate-associated osteonecrosis of the jaw: technical report and follow up of 21 patients. Journal of cranio-maxillofacial surgery: official publication of the European Association for Cranio-Maxillo-Facial Surgery, 40(8), 719-725.

Voss, P. J., Matsumoto, A., Alvarado, E., Schmelzeisen, R., Duttenhöfer, F., \& Poxleitner, P. (2017). Treatment of stage II medication-related osteonecrosis of the jaw with necrosectomy and autologous bone marrow mesenchymal stem cells. Odontology, 105(4), 484-493.

Watts N. B. (2003). Bisphosphonate treatment of osteoporosis. Clinics in geriatric medicine, 19(2), 395-414. 